\title{
Analysis of the BEAVRS PWR benchmark using SCALE and PARCS*
}

Piotr Darnowski, Michat Pawluczyk

\begin{abstract}
This paper presents an analysis of the Benchmark for Evaluation And Validation of Reactor Simulations (BEAVRS) performed using SCALE 6.1.2 and PARCS 3.2 computer codes. The benchmark specification contains a detailed design, operational data and measurements for a real 4-loop Westinghouse pressurized water reactor (PWR). The lattice physics simulations were prepared using TRITON depletion sequence and NEWT neutron transport solver (SCALE package). The 238-neutron group library based on evaluated nuclear data file - ENDF/B-VII nuclear data libraries was applied. A set of branch and burnup calculations was prepared, and group constants in the form of PMAXS files were generated with GenPMAXS. The full-core models were prepared using the PARCS nodal-diffusion core simulator. The PMAXS libraries were used with PARCS to investigate the core operation. The hot zero power measurement data, including control rod worths and critical boron concentrations, were compared using simulations, and satisfactory results were achieved. The first fuel cycle was simulated, and acceptable agreement with boron letdown curve and measurements were obtained. Finally, conclusions and recommendations for future research were presented.
\end{abstract}

Keywords: BEAVRS • nuclear reactor • PARCS • PWR • SCALE • TRITON

P. Darnowski ${ }^{\bowtie}$, M. Pawluczyk Institute of Heat Engineering Faculty of Power and Aeronautical Engineering Warsaw University of Technology 21/25 Nowowiejska St., 00-665 Warsaw, Poland E-mail: piotr.darnowski@itc.pw.edu.pl

Received: 21 September 2017

Accepted: 21 March 2019

\section{Introduction}

Reactor core simulations are fundamental for economical and safe nuclear power plant operation. Currently, several different simulation tools are available, and nuclear engineers perform calculations to predict the reactor core state. This work is an example of contemporary computational tools applied to perform reactor simulations of a large pressurized water reactor (PWR).

The SCALE-PARCS two-step methodology was selected by the National Atomic Energy Agency (PAA), a Polish regulatory body, as an approach to nuclear reactor safety research. It played an essential role in the assessment of the first Polish NPP. The SCALE-PARCS approach was not tested in Poland a priori to the project (in 2015) reported in this paper. This work was supported by PAA as a part of the effort to assess the methodology. Moreover, it was a part of the training and experience-gathering process to enhance reactor safety competencies.

\footnotetext{
* This paper is based on an oral presentation showed at NUTECH-2017 Conference, 10-13 September 2017, Kraków, Poland.
} 

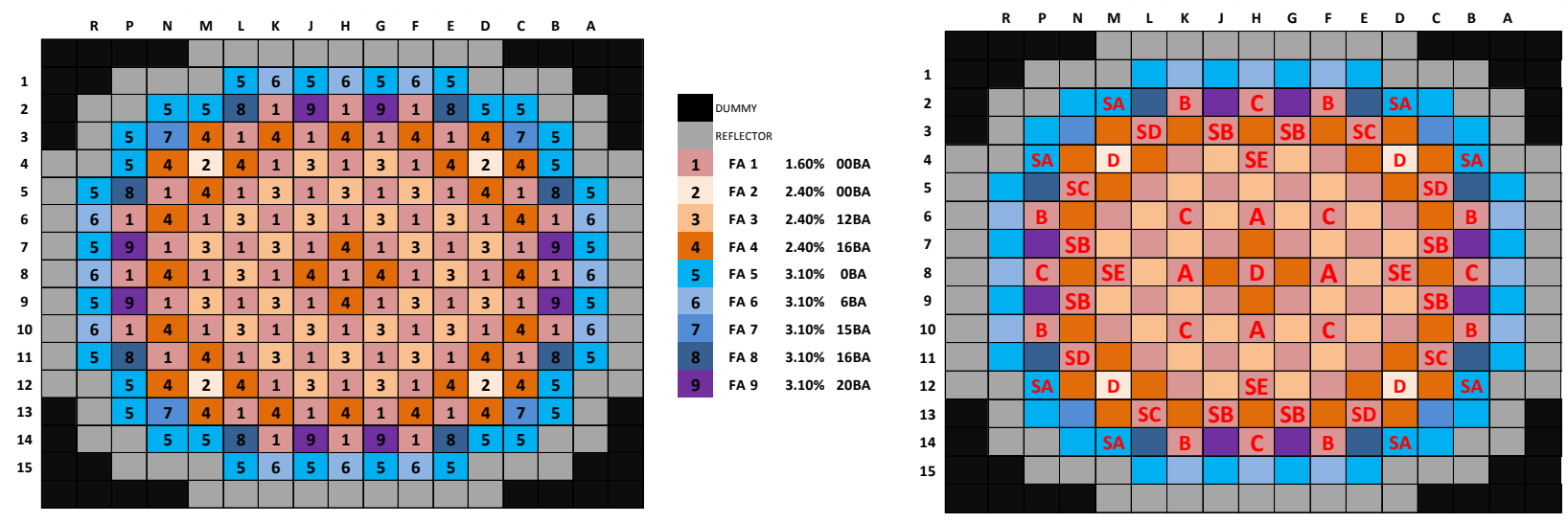

Fig. 1. The BEAVRS core. The left-hand side presents the core loading pattern for Cycle 1, and the right-hand side presents control banks pattern.

The primary aim of this work was to perform tests and assessments of the SCALE-PARCS two-step methodology with a full-core benchmark problem based on real plant data. Additionally, development and verification of the PWR nuclear reactor mathematical model was also a goal. Clearly, Benchmark for Evaluation and Validation of Reactor Simulations (BEAVRS) is only a single benchmark and its solution is only a portion of the larger testing and assessment effort. It is worth mentioning that the presented work was also a part of the effort to develop methodology and computational tools necessary to predict detailed core inventory dependent on space and time.

In the first step, the SCALE package with TRITON sequence and NEWT transport solver [1] were applied for the lattice physics calculations and fuel burnup. Afterwards, TRITON output was processed using GenPMAXS tool, which allowed to generate a series of few-group constant cross-section libraries (PMAXS) for the 3D coarse-mesh nodal-diffusion core simulator PARCS [2].

The SCALE-PARCS two-step methodology applied in this work is similar to the US Nuclear Regulatory Commission's (USNCR) approach to reactor safety research. It is also in use by other institutions worldwide. The methodology performance has a potential impact on $\sim 100$ reactors - one-fourth of world nuclear fleet. Hence, it is as an essential task to perform constant validation and verification of the code and methodology.

The BEAVRS was published in 2013 by the Massachusetts Institute of Technology (MIT) Computational Reactor Physics Group, and it was updated several times [3-5]. Its purpose is to allow comparison of various reactor physics computer codes applied to the full-core calculations with real plant data. The benchmark specification contains a detailed description of an unknown (real) nuclear power plant with a 4-loop PWR 3411 MWth Westinghouse reactor, which is located in the USA. The documentation contains details of the hot zero power (HZP) measurements and reactor operating conditions for the first and the second fuel cycles.

Several research reports on BEAVRS solutions were published for a large variety of simulation methodologies [6-23]. The model developed in this work was compared with available benchmark data for the first fuel cycle presented in publications [8, 24]. The SCALE-PARCS two-step sequence was tested using BEAVRS data only in a single publicly available conference report, PHYSOR-2018, which describes the University of Illinois at Urbana-Champaign (UIUC) research financed by USNRC [23]. These results were performed using newer SCALE 6.2 .2 , and, however, this work was performed using SCALE 6.1.2.

\section{Core models}

For the first fuel cycle, there are nine types of fuel assemblies with $17 \times 17$ lattice, and they are modelled as separate 2D TRITON models for lattice physics calculations. The fuel has three enrichments, 1.6, 2.4 and $3.1 \mathrm{wt} \%$, with a different population of burnable absorber (BA) in the form of borosilicate rods: 0, 6, 12, 15, 16 and 20 (see Fig. 1, left). Detailed fuel and core design data are available in [3-5], and they will not be reported in this paper.

Two additional models for the axial and radial reflectors were prepared. The SCALE/TRITON guidelines for reflector simulations prepared by the Oak Ridge National Laboratory (ORNL) were utilized [25]. In order to simulate radial reflector, the assembly FA5 was applied with the additional square region having the same size as an assembly. It was filled with three regions: steel (core barrel), water (downcomer) and steel (vessel). In the case of the axial reflector, the most abundant FA1 assembly was applied with a rectangular region filled with a homogenous mixture of water and structural materials. Moreover, for the sake of simplicity and to reduce computational effort, spacer grid models were not prepared.

The average specific power for burnup calculations was equal to $41.7 \mathrm{MWth} / \mathrm{tHM}$. The TRITON burnup steps were selected following the code recommendations described in [25] where smaller steps were applied for the fuel with BAs. Smaller steps are necessary to accurately calculate burnable absorbers evolution for the time interval with high BAs concentrations. For the fuel with BAs burnup 


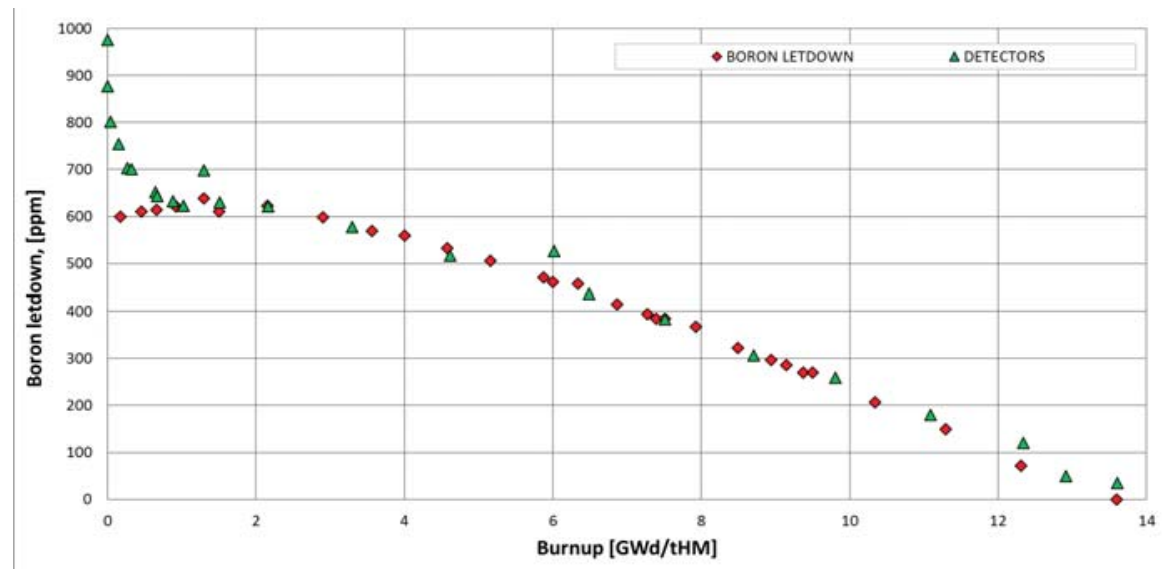

Fig. 2. BEAVRS Cycle 1 power operation datasets [4].

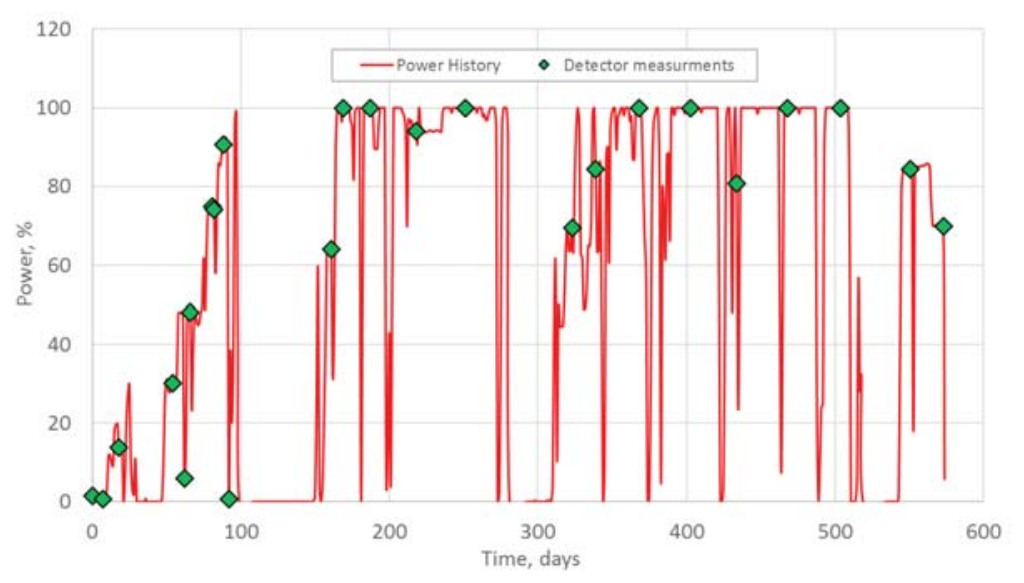

Fig. 3. BEAVRS Cycle 1 power history with indicated detector measurements. Based on [4].

scheme, expressed in time intervals (effective full power days, EFPDs), were: 5, 15, 7, 25 (7 steps), 40 (22 steps) days respectively and summing up to 1075 days (EFPDs) and burnup of $\sim 44.0 \mathrm{GWd} / \mathrm{tHM}$. In the case of assemblies without BAs, time intervals were: 5, 15, 25 (7 steps), 60 (15 steps) days respectively and summing up to 1065 EFPDs with burnup $\sim 44.4 \mathrm{GWd} / \mathrm{tHM}$. In the case of burnup calculations, the boron concentration was set to be constant and equal to the average boron contraction $(378 \mathrm{ppm})$ for the benchmarks' letdown curve. The hot full power (HFP) state has moderator density equal to $0.71 \mathrm{~g} / \mathrm{cc}$, fuel temperature equal to $900 \mathrm{~K}$ and moderator temperature equal to $580 \mathrm{~K}$. What is more, the HFP state is also a reference state for the branch calculations dedicated to the power operation. The HZP reference state for the TRITON models and branches was different with moderator density $0.74 \mathrm{~g} / \mathrm{cc}$, boron inventory $975 \mathrm{ppm}$ and all temperatures equal to $566 \mathrm{~K}$. Branch details are presented in the next sub-section.

The PARCS core model has the same radial nodalization as the core loading pattern (Fig. 1), one node per one fuel assembly and totally 257 nodes. Axial nodalization consists of 20 equally length active core axial levels and two reflector nodes, top and bottom. The BEAVRS specification contains two different fuel cycle datasets (see Fig. 2) and two different PARCS burnup schemes were developed. For the boron letdown calculations (100\% HFP for 327.2 EFPDs), a relatively dense burnup scheme was applied with points (days) coherent with the benchmarks' boron letdown values. Otherwise, the burnup scheme for detailed calculations with variable parameters was based on the plant power history (see Fig. 3). The final calculations were performed with burnup scheme based on the UIUC work [23]. The basic approach was to obtain proper burnup, as measured at a given point in time.

The models used to obtain the results presented in this report are based on the specification available at the time of the research project - revision 1.1.1 [3]. It is necessary to mention that the benchmark revision 2.0.1 was published in 2017 [4] and 2.0.2 in 2018 [26]. They have some minor updates in comparison to revision 1.1.1. The results presented in this work were compared with revision 2.0.1 data.

\section{Branches}

Two different branch patterns were prepared for this study. The first branch is dedicated only to the HZP state, and it is a large branch covering large phase space of potential reactor states. It is also characterized by a different reference state. It has three different boron concentrations, all possible control rod branches and two fuel temperature branches. Calculations were performed without fuel burnup (Table 1) resulting in a substantial reduction of computational time. 
Table 1. Branch dedicated to hot zero power calculations

\begin{tabular}{|c|c|c|c|c|c|}
\hline $\begin{array}{l}\text { Number } \\
\text { units }\end{array}$ & $\begin{array}{c}\text { Control rods } \\
{[1-\text { in, } 0-\text { out }]}\end{array}$ & $\begin{array}{c}\text { Moderator } \\
\text { density } \\
{[\mathrm{g} / \mathrm{cc}]}\end{array}$ & $\begin{array}{c}\text { Boron } \\
\text { concentration } \\
{[\mathrm{ppm}]}\end{array}$ & $\begin{array}{c}\text { Fuel } \\
\text { temperature } \\
{[\mathrm{K}]}\end{array}$ & $\begin{array}{c}\text { Moderator } \\
\text { temperature } \\
{[\mathrm{K}]}\end{array}$ \\
\hline 0 & 0 & 0.74 & 975 & 566 & 566 \\
\hline 1 & 0 & 0.71 & 975 & 566 & 566 \\
\hline 2 & 1 & 0.74 & 975 & 566 & 566 \\
\hline 3 & 1 & 0.71 & 975 & 566 & 566 \\
\hline 4 & 0 & 0.74 & 1500 & 566 & 566 \\
\hline 5 & 0 & 0.71 & 1500 & 566 & 566 \\
\hline 6 & 1 & 0.74 & 1500 & 566 & 566 \\
\hline 7 & 1 & 0.71 & 1500 & 566 & 566 \\
\hline 8 & 0 & 0.74 & 0 & 566 & 566 \\
\hline 9 & 0 & 0.71 & 0 & 566 & 566 \\
\hline 10 & 1 & 0.74 & 0 & 566 & 566 \\
\hline 11 & 1 & 0.71 & 0 & 566 & 566 \\
\hline 12 & 0 & 0.71 & 975 & 900 & 566 \\
\hline 13 & 1 & 0.71 & 975 & 900 & 566 \\
\hline 14 & 0 & 0.71 & 1500 & 900 & 566 \\
\hline 15 & 1 & 0.71 & 1500 & 900 & 566 \\
\hline 16 & 0 & 0.71 & 0 & 900 & 566 \\
\hline 17 & 1 & 0.71 & 0 & 900 & 566 \\
\hline 18 & 0 & 0.74 & 975 & 900 & 566 \\
\hline 19 & 1 & 0.74 & 975 & 900 & 566 \\
\hline 20 & 0 & 0.74 & 1500 & 900 & 566 \\
\hline 21 & 1 & 0.74 & 1500 & 900 & 566 \\
\hline 22 & 0 & 0.74 & 0 & 900 & 566 \\
\hline 23 & 1 & 0.74 & 0 & 900 & 566 \\
\hline
\end{tabular}

Table 2. Branch dedicated to power operation

\begin{tabular}{|c|c|c|c|c|c|}
\hline $\begin{array}{l}\text { Number } \\
\text { units }\end{array}$ & $\begin{array}{l}\text { Control rods } \\
{[1-\text { in, } 0-\text { out }]}\end{array}$ & $\begin{array}{c}\text { Moderator } \\
\text { density } \\
{[\mathrm{g} / \mathrm{cc}]}\end{array}$ & $\begin{array}{c}\text { Boron } \\
\text { concentration } \\
{[\mathrm{ppm}]}\end{array}$ & $\begin{array}{c}\text { Fuel } \\
\text { temperature } \\
{[\mathrm{K}]}\end{array}$ & $\begin{array}{c}\text { Moderator } \\
\text { temperature } \\
{[\mathrm{K}]}\end{array}$ \\
\hline 0 & 0 & 0.71 & 378 & 900 & 580 \\
\hline 1 & 0 & 0.74 & 378 & 900 & 580 \\
\hline 2 & 1 & 0.71 & 378 & 900 & 580 \\
\hline 3 & 1 & 0.74 & 378 & 900 & 580 \\
\hline 4 & 0 & 0.71 & 0 & 900 & 580 \\
\hline 5 & 0 & 0.74 & 0 & 900 & 580 \\
\hline 6 & 1 & 0.71 & 0 & 900 & 580 \\
\hline 7 & 1 & 0.74 & 0 & 900 & 580 \\
\hline 8 & 0 & 0.71 & 756 & 900 & 580 \\
\hline 9 & 0 & 0.74 & 756 & 900 & 580 \\
\hline 10 & 1 & 0.71 & 756 & 900 & 580 \\
\hline 11 & 1 & 0.74 & 756 & 900 & 580 \\
\hline 12 & 0 & 0.69 & 378 & 900 & 580 \\
\hline 13 & 1 & 0.69 & 378 & 900 & 580 \\
\hline 14 & 0 & 0.69 & 0 & 900 & 580 \\
\hline 15 & 1 & 0.69 & 0 & 900 & 580 \\
\hline 16 & 0 & 0.69 & 756 & 900 & 580 \\
\hline 17 & 1 & 0.69 & 756 & 900 & 580 \\
\hline 18 & 0 & 0.71 & 975 & 900 & 580 \\
\hline 19 & 0 & 0.74 & 975 & 900 & 580 \\
\hline 20 & 0 & 0.74 & 975 & 566 & 580 \\
\hline 21 & 1 & 0.71 & 378 & 566 & 580 \\
\hline
\end{tabular}

The second branch configuration (Table 2) is dedicated for a power operation with fuel burnup. In comparison to the HZP branch, it covers phase space corresponding to the potential operational states. It is worth mentioning that several alternative branches were created during the model development and it was the most time-consuming part of the project. All branch cases were calculated with a single moderator temperature as it is the common practice in this type of analysis [23, 27, 28]. Model 


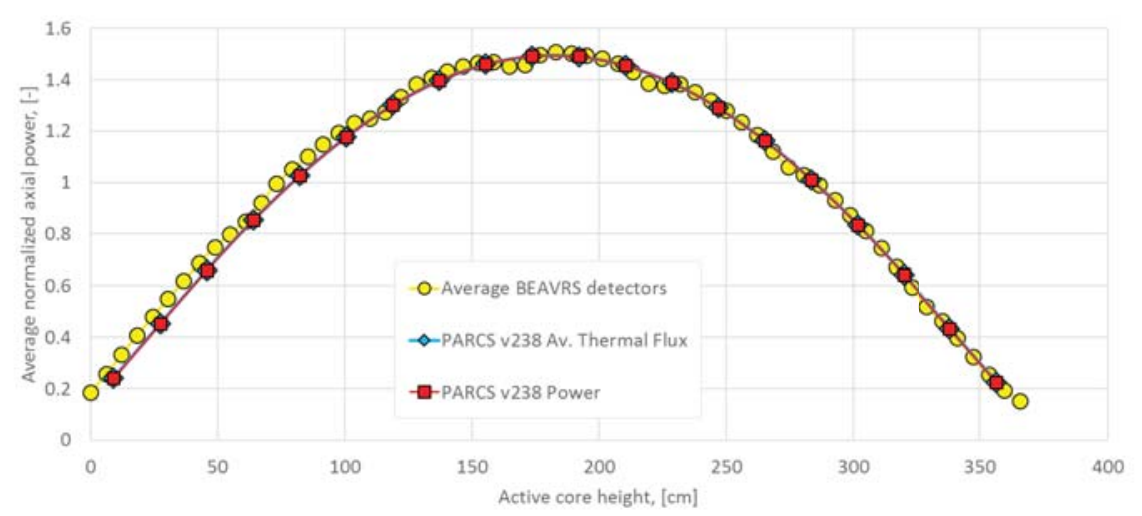

Fig. 4. Comparison of the BEAVRS radially averaged flux measurements (yellow dots) with PARCS radially averaged power (red) and thermal flux distribution (blue) [4].

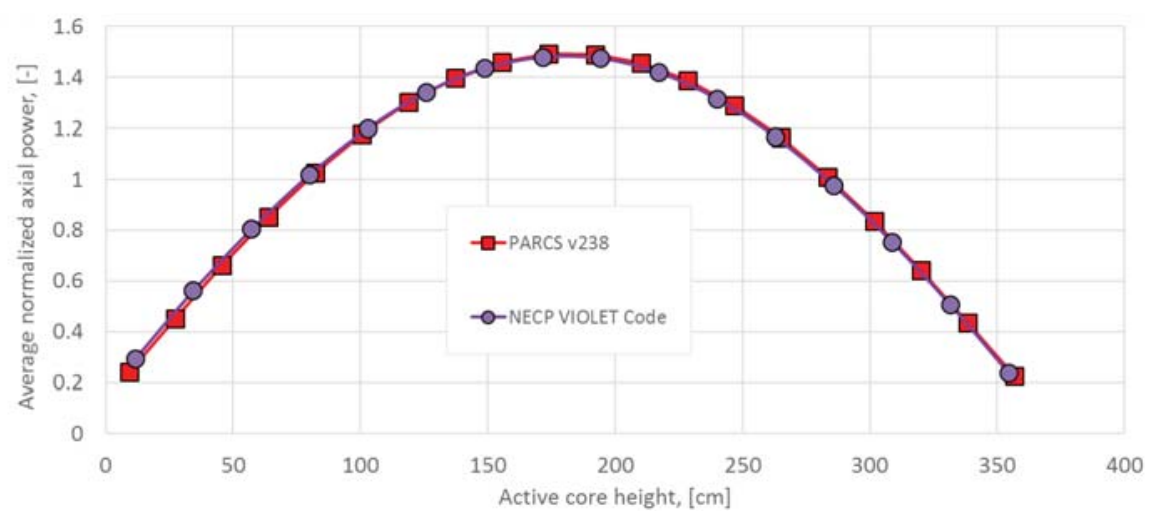

Fig. 5. Comparison of the PARCS radially averaged axial power distribution with axial power distribution calculated with NECP code [8].

development guidelines described in [25] were applied as far as possible.

\section{Results and discussion}

\section{Hot zero power (HZP)}

Figure 4 presents the axial distribution of radially averaged detector measurements (yellow circles) provided with BEAVRS specification compared with PARCS calculated axial thermal flux and axial power radially averaged distributions. A reasonable agreement was obtained with a slight underestimation of the flux (lower leakage) at the bottom of the core. The reason is the bottom reflector modelling as the same model was used for the top and bottom reflector. It is also possible to observe the lack of small flux depressions due to the absence of spacer grid modelling.

The axial power distribution calculated with PARCS and reference solution obtained with NECP-VIOLET code ([8]) is compared in Fig. 5. In this case, one can also observe slight underestimation of the PARCS power distribution, and it is due to the same reasons as in the case of the flux distribution, as the power distribution is strongly related to the thermal flux distribution.

The left-hand side of Fig. 6 presents the axially averaged radial thermal flux distribution calculated with PARCS compared with measurements presented in the BEAVRS specification. The BEAVRS data was corrected for a tilt effect discussed in the revision 2.0.1 [4]. The obtained root mean squared error (RMSE) for the thermal flux and BEAVRS is $\sim 5 \%$. Underestimation of the flux inside the core was observed with the highest relative difference (RD) value equal to $-9.5 \%$. Otherwise, an overestimation was observed for the outer boundary of the core with the highest difference $+7.8 \%$. The obtained deviations are not substantially different from the alternative results available in the literature $[8,22,23]$. For example in UIUC paper [23], they obtained RMSE equal to $5.38 \%$ in comparison to our value $4.89 \%$ (see Fig. 6). The reason for these differences is the radial reflector modelling and limited capabilities of the SCALE-PARCS sequence. During test calculations, not reported in this paper, a strong influence of the radial reflector model was observed with opposite effect as in Fig. 4 - underestimation of the flux at the boundary and overestimation at the centre of the core. Similar effects were also observed during studies of different PWR cores [29].

Higher flux at the vicinity of the reflector leads to higher leakage reduction of the $k$-eff. On the contrary, higher flux at the outer ring increases the fission reaction rate, as the fuel has high enrichment in that region and causes an increase of the neutron multiplication. Otherwise, the reduced flux at the centre of the core results in a reduction of the fission reaction rate in this region. The balance of those effects contributes to the reduction of the neutron multiplication of the core, which was observed. Another 

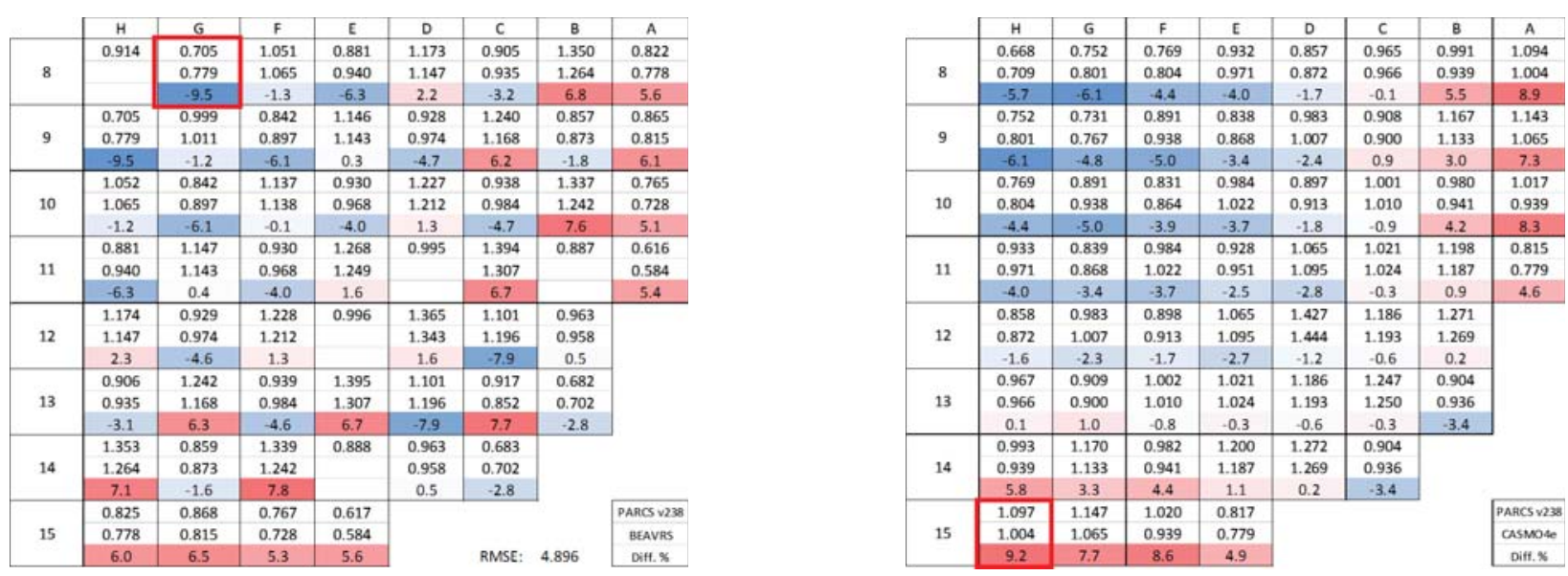

Fig. 6. Left - comparison of the thermal flux distribution calculated with PARCS and tilt corrected detector responses described in BEAVRS specification [4]. Right - comparison of the radial power distribution calculated with PARCS and results obtained with CASMO-4E from Ref. [8].

Table 3. Comparison of the BEAVRS critical boron concentrations [14] with PARCS results for Cycle 1 hot zero power control banks insertion tests

\begin{tabular}{lccc}
\hline \multirow{2}{*}{ Parameter } & \multicolumn{3}{c}{ Critical boron concentration [ppm] } \\
\cline { 2 - 4 } & BEAVRS & V238 groups & Difference [ppm] \\
\hline ARO & 975 & 957 & -18 \\
D in & 902 & 894 & -8 \\
C, D in & 810 & 796 & -14 \\
A, B, C, D in & 686 & 655 & -31 \\
A, B, C, D, SE, SD, SC in & 508 & 480 & -38 \\
\hline
\end{tabular}

ARO - all rods out.

Table 4. Effective multiplication factors calculated for different control rod states for the PARC core with BEAVRS values of boron concentration [4]

\begin{tabular}{lcccc}
\hline \multirow{2}{*}{$\begin{array}{c}\text { Parameter } \\
\text { N }\end{array}$} & $\begin{array}{c}\text { Boron concentration } \\
{[\mathrm{ppm}]}\end{array}$ & BEAVRS & V238 groups & Difference [pcm] \\
\cline { 3 - 5 } & 975 & 1.00000 & 0.99781 & -220 \\
ARO & 902 & 1.00000 & 0.99902 & -98 \\
D in & 810 & 1.00000 & 0.99837 & -164 \\
C, D in & 686 & 1.00000 & 0.99628 & -373 \\
A, B, C, D in & 508 & 1.00000 & 0.99553 & -449 \\
A, B, C, D, SE, SD, SC in & &
\end{tabular}

$\mathrm{ARO}$ - all rods out.

source of uncertainty can be the fact that the detector measurements do not exactly measure thermal fission reaction rate (fission chambers), in the benchmark, there is no explicit description of this issue. The fast flux is minor in comparison to the thermal flux and accounting for it does not produce a significant difference in the results.

The right-hand side of Fig. 6 presents a comparison of the PARCS calculated radial power distribution with the reference solution obtained with CASMO-4E [8]. The effects and deviations, analogous to the thermal flux, were observed. The maximum relative power outer boundary deviation is $\sim 9 \%$, and the maximum inner deviation is $\sim-6 \%$.

The comparison of the BEAVRS and PARCS critical boron inventory is presented in Table 3 . The lowest difference is $-8 \mathrm{ppm}(-1.8 \%)$ for the case with D-bank inserted and the highest is $-38 \mathrm{ppm}$ $(-7.5 \%)$. Observed results are in agreement with the industrial limit of $50 \mathrm{ppm}$ [30]. Hence, it might be considered a satisfactory result.
Table 4 presents calculations of the multiplication factor for the same states as in critical boron concentration calculations but with boron inventories being equal to the BEAVRS's values. The results are correspondent to Table 3 but expressed in terms of reactivity, again showing similar differences. The ARO (all rods out) case deviation is $220 \mathrm{pcm}$, and it is satisfactory value. The highest deviation, for the A, B, C, $\mathrm{D}, \mathrm{SE}, \mathrm{SD}, \mathrm{SC}$ case is $\sim 450 \mathrm{pcm}$, and it is a relatively large value. It can be observed, that the results deviation increases with the increase of the control banks population inserted into the core (Tables 3 and 4).

Control rod banks worth results are presented in Table 5. Banks pattern is visible in Fig. 1(right). All PARCS calculations were performed with $975 \mathrm{ppm}$ of boron. The highest difference was obtained with A, D, C, B banks inserted, and the difference is about $-31 \%(-174 \mathrm{pcm})$, otherwise the lowest difference is $-3.3 \%(-26 \mathrm{pcm})$ for D-bank inserted only. The maximum difference may be considered as moderate. The differences 
Table 5. Comparison of the BEAVRS control rod bank worth [4] with PARCS results

\begin{tabular}{lccc}
\hline \multirow{2}{*}{ Parameter all@ 975 ppm boron } & \multicolumn{3}{c}{ Control rod bank worths [pcm] } \\
\cline { 2 - 4 } & BEAVRS & V238 groups & Difference [pcm] \\
\hline D in & 788 & 762 & -26 \\
C with D in & 1203 & 1149 & -54 \\
B with D, C in & 1171 & 1303 & 132 \\
A with D, C, B in & 548 & 374 & -174 \\
SE with D, C, B, A in & 461 & 342 & -119 \\
SD with D, C, B, A, SE in & 772 & 746 & -26 \\
SC with D, C, B, A, Se, SD in & 1099 & 974 & -125 \\
\hline
\end{tabular}

can be explained by investigation of banks loading pattern and thermal flux measurements. It can be observed that the difference is low regarding reactivity when the control banks are inserted into the region of the core with more accurate flux prediction. For example, the case with only D-bank inserted (see Fig. 1, i.e. location D-12) has a flux difference equal to only $1.6 \%$. Otherwise, insertion of bank B in the B, D, C cases introduces overestimation of the worth even if the control rod worth (CRW) for D and C was underestimated. The thermal fluxes in positions of B-bank (i.e. F-14, B-10) are overestimated, and control rod effects are stronger than in the BEAVRS case.

The obtained HZP results (Tables 3-5) are considered as satisfactory in the framework of the research project, especially considering the applied model simplifications and the applied computational tools.

\section{Fuel cycle results}

In general, the so-called boron letdown curve is a measure of the excess reactivity of the core, and for the BEAVRS, it was calculated for 327.2 days (EFPDs) at $100 \%$ full power with all control rods removed. The comparison of the boron letdown curves for PARCS, BEAVRS [4] and reference Serpent-ARES (high accuracy) results prepared by Finnish VTT [24] is presented in Fig. 7.

An excellent agreement was observed for PARCS (Fig. 7) for burnup lower than $6 \mathrm{GWd} / \mathrm{tHM}$; oth- erwise, results are slowly diverging with the final deviation being less than $30 \mathrm{ppm}$ at the end of the cycle (EOC). All results are characterized by a substantial margin to $50 \mathrm{ppm}$ limit and are satisfactory.

The comparison of the PARCS results with BEAVRS boron concentration measurements performed at the time of detector measurements is presented in Fig. 8. The case was calculated with xenon and samarium transient option, on the contrary to the boron letdown, which was calculated for equilibrium conditions. The most significant difference was observed for an operating point near the end of the first outage. It was the only point with a deviation higher than the 50-ppm limit. Otherwise, during and after outages, large variations are possible as poison transients are present. All other results are within the limit with about $10 \mathrm{ppm}$ difference at the beginning of cycle (BOC). The EOC boron concentration difference to the BEAVRS is about 30 ppm (Fig. 8). The observed agreement is satisfactory, especially considering complex conditions, variable power operation with boron and control rods manoeuvring and three outages (Fig. 2).

\section{Conclusions}

The HZP state and the first fuel cycle of the BEAVRS PWR were simulated using PARCS 3.2 core simulator and SCALE 6.1.2 neutronics package with TRITON-NEWT sequence. The applied methodology and

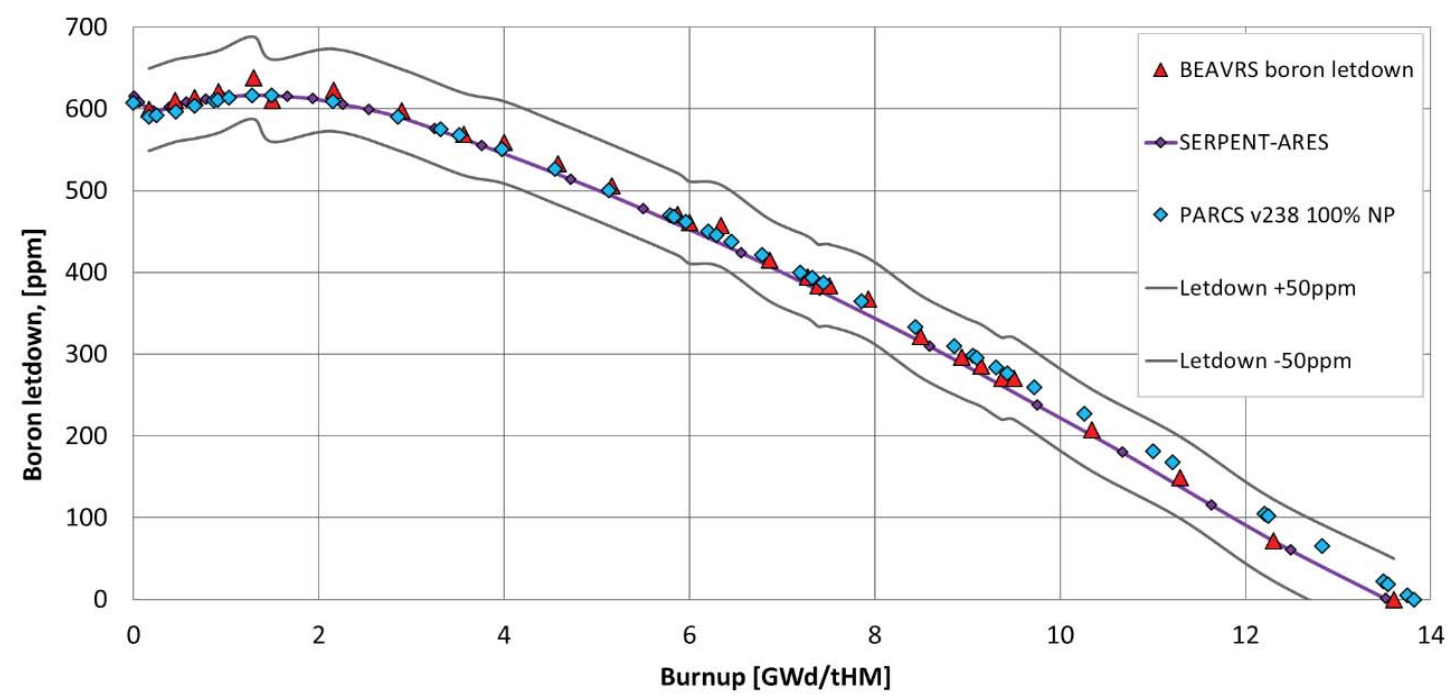

Fig. 7. Comparison of the BEAVRS boron letdown curve [4] with PARCS branch case 1 and case 2 and state-of-the-art results obtained by VTT and reported in [24]. 


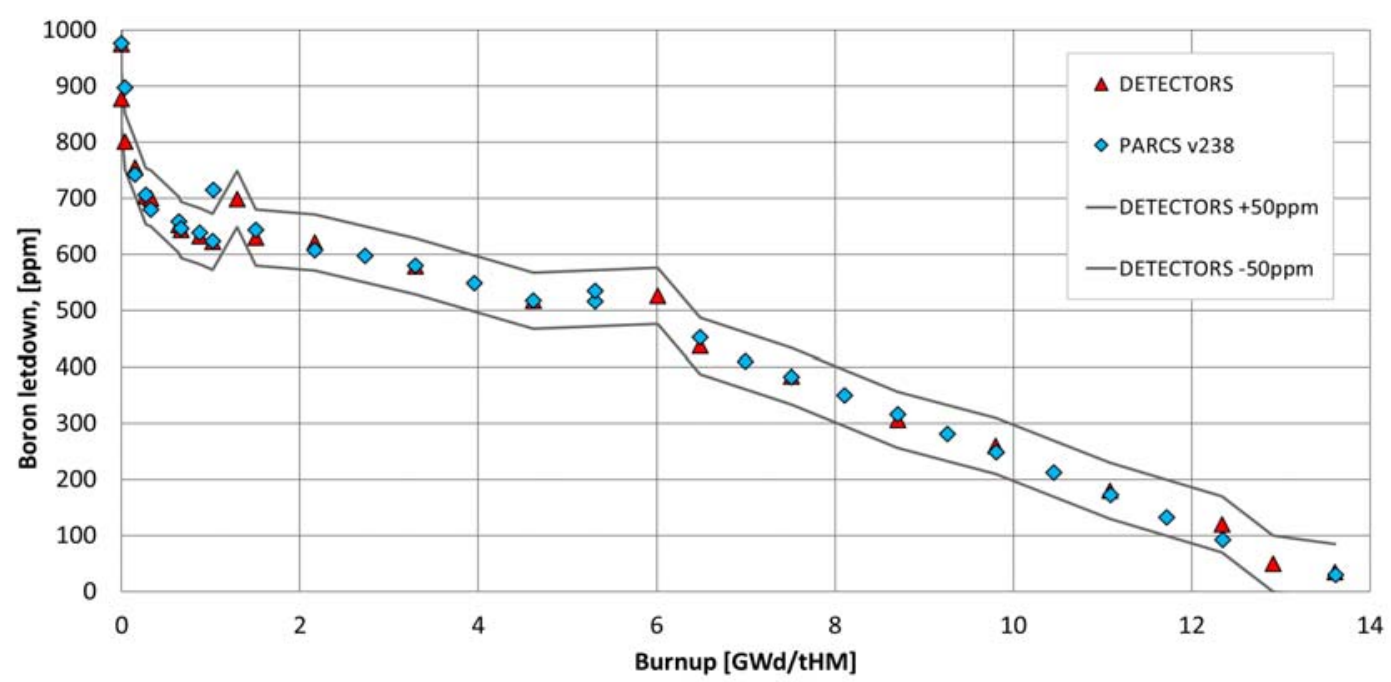

Fig. 8. Comparison of the boron measurements [4] performed during Cycle 1 with PARCS calculations.

codes were tested against the benchmark, and acceptable results were obtained. It can be concluded that the SCALE-PARCS two-step methodology is an appropriate and effective tool for PWR reactor fuel cycle simulations and nuclear safety assessment.

This work is a part of the effort to test and assess SCALE-PARCS two-step sequence for regulatory research applications, and it has significance for the Polish NPP programme. Clearly, simulations of single benchmark solution do not allow to assess the sequence completely, but it is an important step.

The satisfactory HZP results were obtained with deviations within industrial limits. The fuel cycle calculations are considered satisfactory. The observed EOC differences for boron letdown curve and detailed calculation based on the measurements were less than $30 \mathrm{ppm}$.

The calculations were performed using 238-neutron groups library (ENDF-VII). It is worth mentioning that similar calculations were also performed with an older 44 neutron group library (ENDF-V), and obtained results were characterized by a significant difference to the benchmark. Hence, they were not reported in this paper.

Several different alternative branches were developed during the research. The presented two branches fulfilled their role, but it is believed that they can be merged into a single, more straightforward branch. Alternatively, more detailed branches can be developed for more violent transients. Otherwise, the branch calculations and development were the most time-consuming elements of the project. Moreover, it may be considered to enhance the models for both HZP and HFP in the course of future activities. Especially, studies of spacer grids effects and reflector modelling improvement should be considered.

Dozens of alternative BEAVRS results are available in literature. Some of them are more accurate solutions obtained with high-fidelity methods like Monte Carlo; other results are less accurate: all were obtained with different computational tools and methodologies $[8,16,19,22,24]$. Only a single report describing BEAVRS simulations using PARCS-SCALE is publicly available [23]. Their results were obtained using
PARCS 3.2 - SCALE 6.2.2 and 56-group ENDF/B-VII library. Considering the importance of the two-step sequence, a single reference solution can be considered as insufficient. This paper provides comparison data obtained using older SCALE 6.1.2, PARCS 3.2 and ENDF/B-VII 238 group library. It can be a valuable reference for researchers studying BEAVRS benchmark with different computer codes.

It can be concluded that the BEAVRS benchmark is a unique test to validate PWR reactor simulations and should be used as a standard test for software testing in the future reactor safety research.

Finally, this work is a first verification of the PWR model, which will be applied as a reference reactor for further nuclear safety research. It is planned to apply it as an NPP model in the development of core inventory prediction tools and in-core fuel management computational tools.

Acknowledgments. This research was financed by the National Atomic Energy Agency (PAA) under the contract $13 / 2015 / \mathrm{DBJ}$ in 2015 . Activities performed in 2017 were financed by the Faculty of Power and Aeronautical Engineering Dean's grant no. 504/03264: "Development of the novel core inventory calculation methodology for the PWR reactor". Extraordinary thanks go to Professor Tomasz Kozlowski for constructive discussions. The authors would like to acknowledge the National Atomic Energy Agency for support. Especially, thanks go to Mr. Szymon Suchcicki and Dr Ernest Staron for very fruitful cooperation. The computer infrastructure used to prepare this publication was provided by "Information Platform TEWI" Project which was funded by the European Union in the framework of the European Social Fund (2007-2013).

\section{References}

1. Oak Ridge National Laboratory. (2011). SCALE: A comprehensive modeling and simulation suite for nuclear safety analysis and design. Version 6.1. Oak Ridge, TN, USA: ORNL. (ORNL/TM-2005/39). 
2. US Nuclear Regulatory Commission. (2013). SCALE/ TRITON Primer: A primer for light water reactor lattice physics calculations using SNAP. U.S. NCR.

3. MIT Computational Reactor Physics Group. (2013). BEAVRS - benchmark for evaluation and validation of reactor simulations. Rev. 1.1.1. Cambridge, UK: MIT CRPG.

4. MIT Computational Reactor Physics Group. (2017). BEAVRS - benchmark for evaluation and validation of reactor simulations. Rev. 2.0.1. Cambridge, UK: MIT CRPG.

5. Horelik, N., Herman, B. R., Forget, B., \& Smith, K. (2013). Benchmark for Evaluation and Validation of Reactor Simulations (BEAVRS). In International Conference on Mathematics and Computational Methods Applied to Nuclear Science and Engineering, May 5-9, 2013, Sun Valley, ID, USA (Vol., 4, pp. 2986-2999). LaGrange Park, IL, USA: American Nuclear Society. (CD edition)

6. Park, H. J., Lee, H. C., Shim, H. J., \& Cho, J. Y. (2016). Real variance analysis of Monte Carlo eigenvalue calculation by McCARD for BEAVRS benchmark. Ann. Nucl. Energy, 90, 205-211. doi: 10.1016/j. anucene.2015.12.009.

7. Liu, S., Liang, J., Wu, Q., Guo, J., Huang, S., Tang, X., Li, Z., \& Wang, K. (2017). BEAVRS full core burnup calculation in hot full power condition by RMC code. Ann. Nucl. Energy, 101, 434-446. doi: 10.1016/j. anucene.2016.11.033.

8. Wan, C., Cao, L., Wu, H., \& Shen, W. (2017). Uncertainty analysis for the assembly and core simulation of BEAVRS at the HZP conditions. Nucl. Eng. Des., 315, 11-19. doi: 10.1016/j.nucengdes.2017.02.020.

9. Electric Power Research Institute. (2015). PWR fuel reactivity depletion uncertainty quantification methods validation using BEAVRS Flux Map Data. EPRI.

10. Leppänen, J., Mattila, R., \& Pusa, M. (2014). Validation of the Serpent-ARES code sequence using the MIT BEAVRS benchmark - initial core at HZP conditions. Ann. Nucl. Energy, 69, 212-225. doi: 10.1016/j.anucene.2014.02.014.

11. Gang, L., Dunfu, S., Baoyin, Z., Rui, L., Zehua, H., \& Yuanguang, F. (2016). The application of JMCT to HZP of BEAVRS. In Physics of Reactors 2016 - PHYSOR 2016 - Unifying Theory and Experiments in the 21st Century, May 1-5, 2016, Sun Valley, ID, USA (pp. 4131-4138). LaGrange Park, IL, USA: American Nuclear Society. (CD edition).

12. Bykov, V. (2016). Solution of the BEAVRS benchmark using CASMO-5/SIMULATE-5 Code sequence. In Physics of Reactors 2016 - PHYSOR 2016 - Unifying Theory and Experiments in the 21st Century, May 1-5, 2016, Sun Valley, ID, USA (pp. 0-3). LaGrange Park, IL, USA: American Nuclear Society. (CD edition).

13. Taforeau, J., \& Salino, V. (2016). Analysis of the MIT BEAVRS Benchmark using the DRAGON-5/PARCS code sequence. In Physics of Reactors 2016 - PHYSOR 2016 - Unifying Theory and Experiments in the 21st Century, May 1-5, 2016, Sun Valley, ID, USA (pp. 2940-2949). LaGrange Park, IL, USA: American Nuclear Society. (CD edition)

14. Li, Z., Wu, H., Cao, L., Tian, C., \& Chen, D. (2016). On-line monitoring analysis of BEAVRS benchmark using NECP-ONION. In Physics of Reactors 2016 PHYSOR 2016 - Unifying Theory and Experiments in the 21st Century, May 1-5, 2016, Sun Valley, ID, USA (pp. 3637-3648). LaGrange Park, IL, USA: American Nuclear Society. (CD edition).

15. Harrison, R. D., \& Wheeler, A. F. (2016). Validation of WIMS/PANTHER PWR fuel reactivity depletion using the BEAVRS Benchmark flux map data. In Physics of Reactors 2016 - PHYSOR 2016 - Unifying Theory and Experiments in the 21st Century, May 1-5, 2016, Sun Valley, ID, USA (pp. 2833-2846). LaGrange Park, IL, USA: American Nuclear Society. (CD edition).

16. Wang, K., Liu, S., Li, Z., Wang, G., Liang, J., Yang, F., Chen, Z., Guo, X., Qiu, Y., Wu, O., Guo, J. J., \& Tang, X. (2017). Analysis of BEAVRS two-cycle benchmark using RMC based on full core detailed model. Prog. Nucl. Energy, 98, 1-12. doi: 10.1016/j. pnucene.2017.04.009.

17. Collins, B., \& Godfrey, A. (2015). Analysis of the BEAVRS Benchmark using VERA-Cs. In ANS MC2015 - Joint International Conference on Mathematics and Computation (M\&C), Supercomputing in Nuclear Applications (SNA) and the Monte Carlo (MC) Method, April 19-23, 2015, Nashville, TN, USA. LaGrange Park, IL, USA: American Nuclear Society. (CD Edition) (11 pp.). Available from https://www.casl.gov/sites/default/files/docs/ CASL-U-2015-0183-000.pdf.

18. Park, H. J., Lee, H. C., Cho, J. Y., Shim, H. J., \& Kim, C. H. (2015). Real variance estimation of BEAVRS benchmark in McCARD Monte Carlo eigenvalue calculations. In ANS MC2015 - Joint International Conference on Mathematics and Computation (M\&C), Supercomputing in Nuclear Applications (SNA) and the Monte Carlo (MC) Method, April 19-23, 2015, Nashville, TN, USA (pp. 110-121). LaGrange Park, IL, USA: American Nuclear Society. (CD edition).

19. Ryu, M., Jung, Y. S., Cho, H. H., \& Joo, H. G. (2015). Solution of the BEAVRS benchmark using the nTRACER direct whole core calculation code. J. Nucl. Sci. Technol., 52, 961-969. doi: 10.1080/00223131.2015.1038664.

20. Kelly, D. J., Aviles, B. N., Romano, P. K., Herman, B. R., Horelik, N. E., \& Forget, B. (2014). Analysis of Select BEAVRS PWR benchmark Cycle 1 results using MC21 and OPENMC. In PHYSOR 2014 - The Role of Reactor Physics towards a Sustainable Future, 28 September -3 October 2014, Kyoto, Japan (pp. 1-15).

21. Suzuki, M., \& Nauchi, Y. (2015). Analysis of BEAVRS benchmark problem by using enhanced Monte Carlo Code MVP with Jendl-4.0. In ANS MC2015 - Joint International Conference on Mathematics and Computation (M\&C), Supercomputing in Nuclear Applications (SNA) and the Monte Carlo (MC) Method, April 19-23, 2015, Nashville, TN, USA (pp. 1-11). LaGrange Park, IL, USA: American Nuclear Society. (CD edition).

22. Wang, Z., Wu, B., Hao, L., Liu, H., \& Song, J. (2018). Validation of SuperMC with BEAVRS benchmark at hot zero power condition. Ann. Nucl. Energy, 111, 709-714. doi: 10.1016/j.anucene.2017.09.045.

23. O'Grady, D., Kozlowski, T., \& Hudson, N. (2018). Analysis of the BEAVRS benchmark using the 
TRITON/PARCS/PATHS two-step sequence. In PHYSOR 2018 - Reactors Physics Paving the Way Towards More Efficient Systems, April 22-26, 2018, Cancun, Mexico (pp. 1-11).

24. Leppänen, J., \& Mattila, R. (2016). Validation of the Serpent-ARES code sequence using the MIT BEAVRS benchmark HFP - conditions and fuel Cycle 1 simulations. Ann. Nucl. Energy, 96, 324-331. doi: 10.1016/j. anucene.2016.06.014.

25. US Nuclear Regulatory Commission. (2012). SCALE/ TRITON Primer: A Primer for Light Water Reactor Lattice Physics Calculations - with SNAP. (NUREG/ CR-7041, ORNL/TM-2011/21). Available from https:// www.nrc.gov/docs/ML1233/ML12338A215.pdf.

26. MIT Computational Reactor Physics Group. (2018, April). BEAVRS - Benchmark for evaluation and validation of reactor simulations.. Release rev. 2.0.2. Available from https://crpg.mit.edu/sites/default/files/ css_injector_images_image/BEAVRS_2.0.2_spec.pdf.
27. Oak Ridge National Laboratory. (2011). SCALE/ TRITON Primer: A primer for Light Water Reactor lattice physics calculations - NO SNAP. (NUREG/ CR-7041, ORNL/TM-2011/21).

28. US Nuclear Regulation Commission. (2013). Cross section generation guidelines for TRACE-PARCS. (NUREG/CR-7164, ORNL/TM-2012/518). Available from https://www.nrc.gov/docs/ML1320/ ML13204A296.pdf.

29. Darnowski, P., Ignaczak, P., Obrębski, P., Stępień, M., \& Niewiński, G. (2018). Simulations of the AP1000-based reactor core with SERPENT computer code. Arch. Mech. Eng., LXV, 295-325. doi: 10.24425/124484.

30. New York Power Authority. (2000, January 18). New York Power, Indian Point 3 Nuclear Power Plant Cycle 11 Physics Test Report. Available from https:// www.nrc.gov/docs/ML0036/ML003679481.pdf. 